\section{Analysis of Chua's Circuit with Transmission Line}

Junji Kawata, Yoshifumi Nishio, and Akio Ushida

\begin{abstract}
The purpose of this brief is that, by an application of the method of characteristics, we analyze chaotic phenomena in Chua's circuit with lossy transmission line. The transmission line is replaced by the equivalent lumped circuit and a time-delayed element so that it can be solved efficiently by the Runge-Kutta method. It is found from numerical experiments that the circuit has complicated and interesting chaotic attractors.
\end{abstract}

Index Terms-Chaos, transmission line.

\section{INTRODUCTION}

Chua's circuit is well known as a representative chaotic circuit, and has been studied extensively by many researchers [1]. If the loss of a transmission line is small enough, it can be regarded as a reactive element. Thus, it is possible to replace the parallel $L C$ resonator of Chua's circuit with a transmission line as shown in Fig. 1(a), where $Z_{L}$ is a load impedance. The nonlinear resistor in Chua's circuit is a piecewise linear function as shown in Fig. 1(b) and described by

$$
\begin{aligned}
i_{R}= & m_{2} v_{R}+\frac{1}{2}\left(m_{0}-m_{1}\right)\left[\left|v_{R}+B_{p 1}\right|-\left|v_{R}-B_{p 1}\right|\right] \\
& +\frac{1}{2}\left(m_{1}-m_{2}\right)\left[\left|v_{R}+B_{p 2}\right|-\left|v_{R}-B_{p 2}\right|\right]
\end{aligned}
$$

where $m_{0}, m_{1}, m_{2}$ are the slopes in the each segment of this function, and $B_{p 1}, B_{p 2}$ denote the breakpoints. In this case, we can expect that the circuit will have interesting behavior because it has many types of reflections depending on $Z_{L}$ and the transmission line length. We will call the circuit "Chua's circuit with transmission line."

If the transmission line is lossless and $Z_{L}=0$, the circuit is referred to as the "time-delayed Chua's circuit." Recently, some papers about the time-delayed Chua's circuit have been reported [2]-[6], where the authors assumed $C=0$ and replaced the nonlinear resistor by one with different nonlinear characteristics. Because the time-delayed Chua's circuit with $C=0$ is governed by a one-dimensional difference equation, detailed analysis using the corresponding one-dimensional map is possible. However, in that system we can not observe chaotic phenomena such as those observed in the original Chua's circuit, e.g., Double Scroll attractors. On the other hand, Hosny et al. [7] have proposed an analytical method for solving nonlinear lumped circuits with a lossless transmission line. As an example, chaotic behavior of the time-delayed Chua's circuit is analyzed for the case of $C \neq 0$. The chaotic attractors and the bifurcation diagrams have been also shown by computer simulation. However, the method can not be applied to the analysis of Chua's circuit with lossy transmission line. Our method in this study can be applied to any kind of lumped circuits and transmission lines. It should be noted that for a lossy transmission line the analysis becomes more complicated than that of the lossless line, because the propagation signal is affected by the attenuation.

Now, we will discuss numerical methods for studying chaotic phenomena in Chua's circuit with transmission line. In the last

Manuscript received January 29, 1996; revised September 17, 1996. This paper was recommended by Associate Editor O. Feely.

J. Kawata is with the Department of Information Science and Systems Engineering, Tokushima Bunri University, Okawa 769-21, Japan.

Y. Nishio and A. Ushida are with the Department of Electrical and Electronic Engineering, Tokushima University, Tokushima 770, Japan.

Publisher Item Identifier S 1057-7122(97)04409-7. decade, many approaches have been proposed for simulating highspeed VLSI circuits. Some of them depend on the convolution technique [8]. Although the method is theoretically elegant, it is not so easy to get the accurate transient response, and it seems to be inefficient when the period is very long such as is the case when analyzing chaotic phenomena. The inverse Laplace transformation technique proposed by Nakhla [9] is also elegant. However, the accumulated error may become serious after long periods, so that we can not use the method for our purpose. Note that these timedomain methods can be efficiently applied to the analysis of impulse responses of VLSI circuits. On the other hand, the ideas of the method of characteristics [10]-[12] are based on the reflection theorem for the transmission lines, and the exponential propagation function is replaced by a time-delayed element and lumped circuit by the Padé approximation. Furthermore, the characteristic impedance is also replaced by the equivalent lumped circuit. These lumped circuits are described by $R L$ or $R C$ ladder circuits, so that they can be solved efficiently by the Runge-Kutta method. The equivalent circuit is a reasonable model from the physical point of view, and suitable for the analysis of chaotic circuits with transmission lines. Note that since Chua's circuit has a piecewise linear resistor, we need to apply a linear interpolation technique to get the accurate solution around the breakpoints.

Numerical experiments for the case of $Z_{L}=0$ and $C \neq 0$ are given in Section III for various parameters. It seems that the attractors become more complicated depending on the circuit parameters.

\section{NUMERICAL ANALYSIS}

The generalized method of characteristics for solving lossy transmission lines proposed by Chang [11] is based on Branin [10]. We will now briefly describe this method. A lossy transmission line, as shown in Fig. 2(a), can be characterized by the frequency-domain chain matrix as follows:

$$
\left(\begin{array}{l}
V_{1} \\
I_{1}
\end{array}\right)=\left(\begin{array}{cc}
\cosh \theta & Z_{0} \sinh \theta \\
\left(1 / Z_{0}\right) \sinh \theta & \cosh \theta
\end{array}\right)\left(\begin{array}{l}
V_{2} \\
I_{2}
\end{array}\right)
$$

where $V_{1}, I_{1}$ and $V_{2}, I_{2}$ are the terminal voltages and currents in Fig. 2(a). $Z_{0}$ and $\theta$ are the characteristic impedance and propagation constant given by

$$
Z_{0}=\sqrt{\frac{s L_{d}+R_{d}}{s C_{d}+G_{d}}}, \quad \theta=\sqrt{\left(s L_{d}+R_{d}\right)\left(s C_{d}+G_{d}\right)} l
$$

for line length $l$. The chain matrix is written in the following form:

$$
\begin{aligned}
& V_{1}=+Z_{0} I_{1}+E_{1} \\
& V_{2}=-Z_{0} I_{2}+E_{2}
\end{aligned}
$$

where

$$
\begin{aligned}
& E_{1}=[\exp (-\theta)]\left(2 V_{2}-E_{2}\right) \\
& E_{2}=[\exp (-\theta)]\left(2 V_{1}-E_{1}\right)
\end{aligned}
$$

show the waveform generators for simulating the delay and attenuation of signals. Thus, the transmission line is modeled by the disconnected two-port network shown in Fig. 2(b). The characteristic impedance $Z_{0}$ and the exponential propagation function $e^{-\theta}$ are synthesized by the equivalent lumped circuits by Padé approximation (see [12] for details). Note that the exponential propagation function 


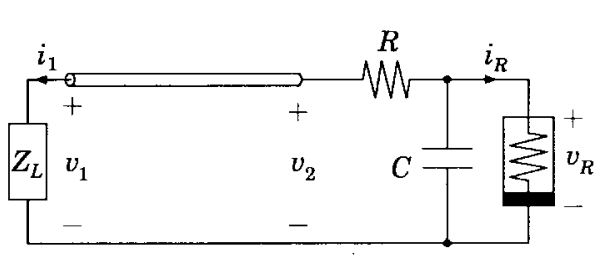

(a)

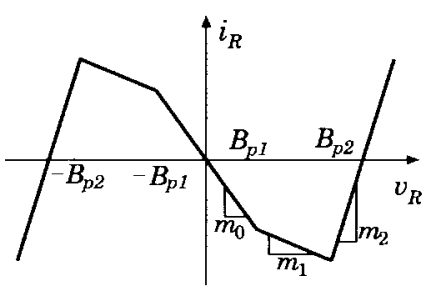

(b)

Fig. 1. (a) Chua's circuit with transmission line. (b) $v-i$ characteristic of nonlinear resistor.

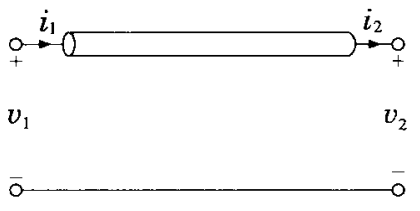

(a)

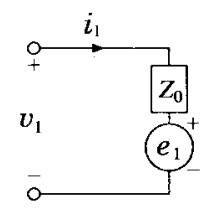

(b)

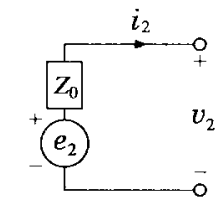

Fig. 2. (a) A transmission line. (b) Its characteristic model.

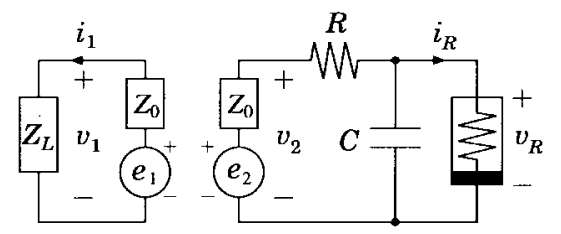

Fig. 3. The equivalent circuit of Chua's circuit with transmission line.

is composed of both an ideal delay line to simulate the time delay and a lumped network to simulate the attenuation loss.

Now, we apply the method of characteristics to Chua's circuit with transmission line. The equivalent circuit is shown in Fig. 3, where the transmission line is replaced by the characteristic model. Thus, we have from (3) and (4) the following circuit equations:

$$
\begin{aligned}
v_{1}(t) & =0 \\
v_{2}(t) & =\frac{R e_{2}(t-\tau)+Z_{0} v_{R}(t)}{R+Z_{0}} \\
C \frac{d v_{R}(t)}{d t} & =\frac{v_{2}(t)-v_{R}(t)}{R}-i_{R}\left(v_{R}\right) \\
e_{1}(t) & =2 v_{2}(t)-e_{2}(t-\tau) \\
e_{2}(t) & =-e_{1}(t-\tau) .
\end{aligned}
$$

Since $Z_{0}$ and $e^{-\theta}$ are modeled by the lumped circuit, the second and last two equations of (5) must be replaced by the corresponding state equations. Thus, the transient responses can be calculated by a numerical integration method such as the Runge-Kutta method. Observe that, since (5) contains the time-delayed waveform generators $e_{1}(t-\tau)$ and $e_{2}(t-\tau)$, their values over the period $\tau$ must be stored. To get the accurate solution, we need to estimate the accurate state variables and the time passing through the breakpoint of piecewise linear resistor. Therefore, we have applied an interpolation technique around the breakpoints. In our example, we used the fourth-order Runge-Kutta method as the numerical integration method.

\section{Simulated Results}

We first simulate Chua's circuit with lossless transmission line by the use of the classical method of characteristics [10]. PeriodDoubling phenomena and Double Scroll attractors similar to those in the original Chua's circuit are observed by the transient analysis. A typical Double Scroll attractor is shown in Fig. 4.

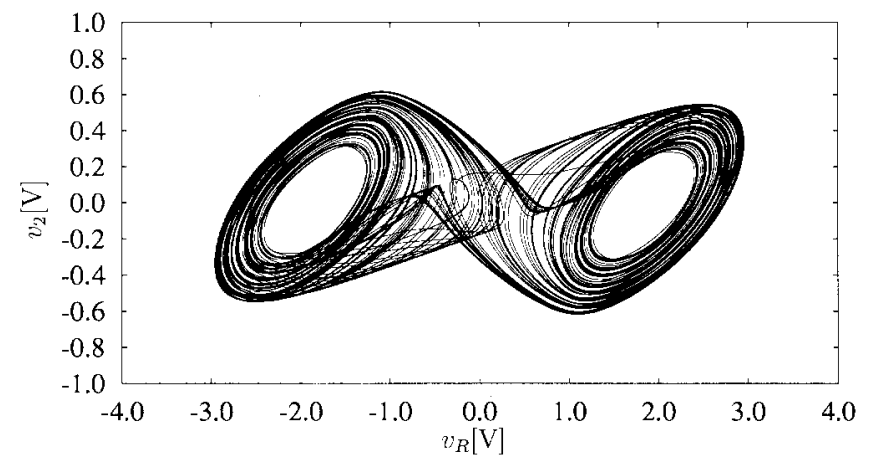

Fig. 4. Attractor of Chua's circuit with lossless transmission line for $R=$ $1690[\Omega]$.

Next, we carry out the simulation for Chua's circuit with lossy transmission line. In the simulation, we have chosen the stepsize small enough, i.e., $\tau / 500$, and used the following parameters:

$$
\begin{aligned}
R_{d} & =0.55[\Omega / \mathrm{m}], \quad L_{d}=0.2375[\mu \mathrm{H} / \mathrm{m}] \\
C_{d} & =95[\mathrm{pF} / \mathrm{m}], \quad G_{d}=0.1[\mathrm{mS} / \mathrm{m}] \\
l & =3[\mathrm{~m}], \quad C=1[\mathrm{pF}]
\end{aligned}
$$

where $R_{d}, L_{d}, C_{d}, G_{d}$ denote the parameters of the lossy transmission line. We have set these parameters by trying to match the chaotic phenomena in real circuits which we will make later. The piecewise linear resistor is symmetric at the origin and the parameters in Fig. 1(b) are given by

$$
m_{0}=-0.9[\mathrm{mS}], \quad m_{1}=-0.5[\mathrm{mS}], \quad B_{p 1}=1[\mathrm{~V}]
$$

where the outer segments with negative slope $m_{1}$ of nonlinear resistor are extended to infinity (i.e., $\pm B_{p 2}= \pm \infty$ ). This transmission line has the ratio $R_{d} C_{d} / L_{d} G_{d}=2.2$ which is approximately distortionless, so we used fourth-order Padé rational function for $Z_{0}$ and $e^{-\theta} .^{1}$ The attractors for different values of the resistor $R$ are shown in Fig. 5. Note that all of the results are obtained for $C \neq 0$. In order to show qualitative difference between two Double Scroll attractors in Figs. 4 and 5(d), we investigated Poincaré map of these attractors. Fig. 6 shows the Poincare map. In the figure the horizontal axis $i_{1}$ is the current flowing through $Z_{L}$ which corresponds to that flowing through the inductor in the original Chua's circuit. As one can see from the Poincaré map in Fig. 6, the attractors of the circuit with lossy transmission line seem more complicated than those of the corresponding circuit with lossless transmission line.

${ }^{1}$ For lossless transmission lines, the characteristic impedance $Z_{0}$ is a constant and the propagation constant $\theta$ is a pure delay, so that the method of characteristics (3) gives the exact solution. On the other hand, for distortionless transmission lines, $Z_{0}$ is also constant and $\theta$ is modeled by a pure delay and a simple resistance circuit without using the Padé approximation, so that (3) also gives the exact solution. Further, it has been reported in [13] that when the parameters of lossy transmission lines satisfy the almost distortionless condition $\left(R_{d} C_{d} / L_{d} G_{d} \approx 1\right)$, the lower order of Padé rational function gives a fairly good approximation. 


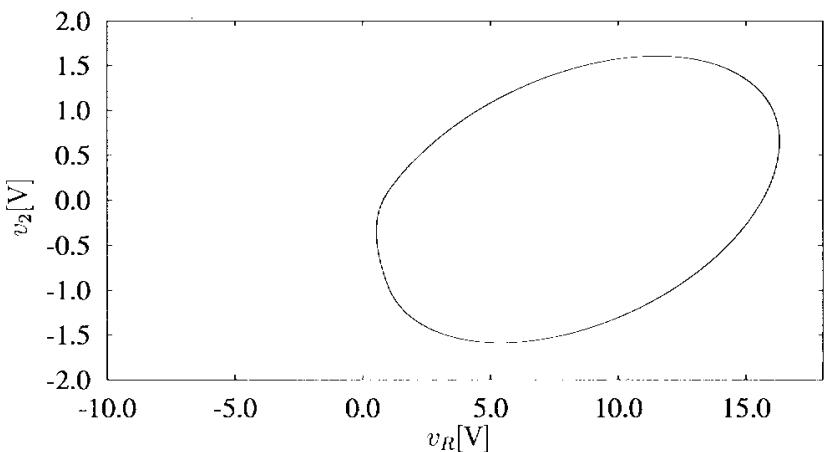

(a)

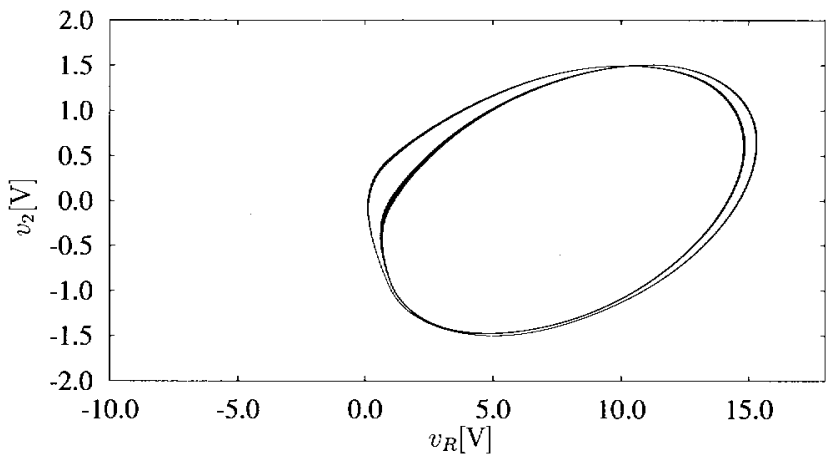

(b)

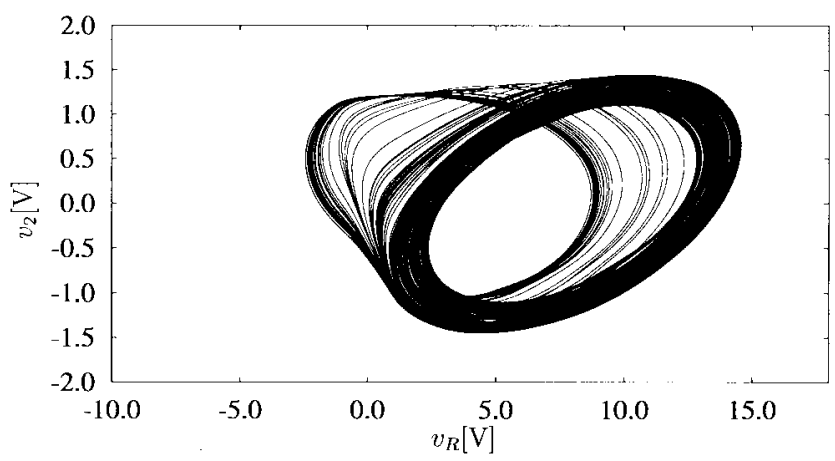

(c)

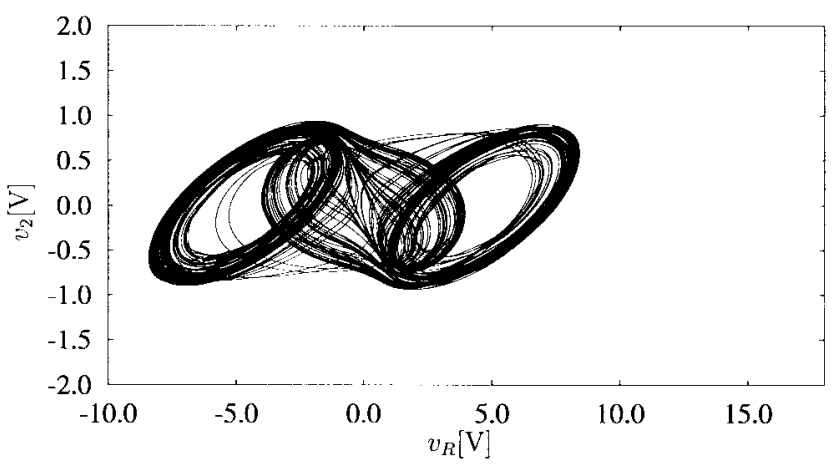

(d)

Fig. 5. Attractors of Chua's circuit with lossy transmission line for (a) $R=1830[\Omega]$. (b) $R=1820[\Omega]$. (c) $R=1810[\Omega]$. (d) $R=1700[\Omega]$.

\section{CONCLUSIONS}

In this brief, we have analyzed Chua's circuit with lossy transmission line for the case of $C \neq 0$ by the method of characteristics and Padé approximation. We have found that Chua's circuit with

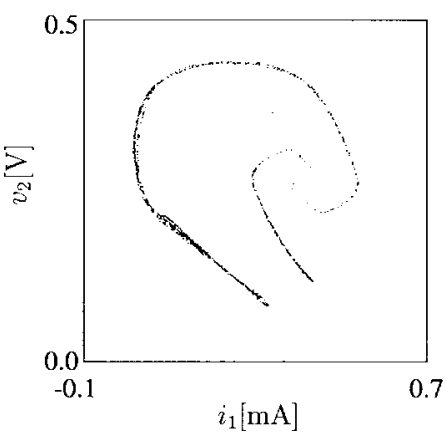

(a)

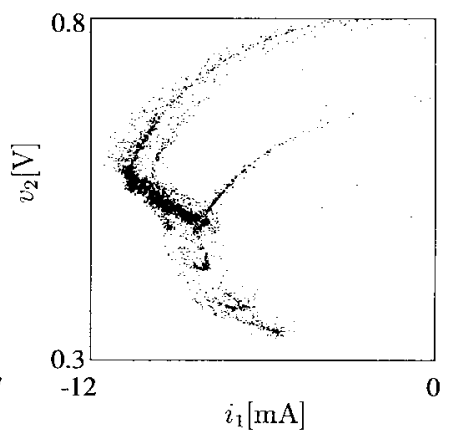

(b)
Fig. 6. Poincaré map. (a) corresponds to Fig. 4 (for the case of $v_{R}=1.5$ [V]). (b) corresponds to Fig. $5(\mathrm{~d})\left(v_{R}=4.0[\mathrm{~V}]\right)$.

transmission line displays various chaotic phenomena including a Double Scroll attractor.

In the future, we are going to simulate this circuit in detail for different circuit parameters, and try to analyze the bifurcation route to chaos and stability of attractors. Chua's circuit with transmission line may also demonstrate different types of chaotic phenomena depending on the line length.

\section{REFERENCES}

[1] L. O. Chua, "Chua's circuit: Ten years later," IEICE Trans. Fundamentals, vol. E77-A, no. 11, pp. 1811-1821, Nov. 1994.

[2] A. N. Sharkovsky et al., "Dry turbulence from a time-delayed Chua's circuit," J. Circuits, Syst., Comput., vol. 3, no. 2, pp. 645-668, June 1993.

[3] Yu. L. Maistrenko, V. L. Maistrenko and L. O. Chua, "Cycles of chaotic intervals in a time-delayed Chua's circuit," Int. J. Bifurc. Chaos, vol. 3, no. 6, pp. 1557-1572, 1993.

[4] A. N. Sharkovsky, "Chaos from a time-delayed Chua's circuit", IEEE Trans. Circuits Syst., vol. 40, pp. 781-783, Oct. 1993.

[5] _ _Ideal turbulence in an idealized time-delayed Chua's circuit," Int. J. Bifurc. Chaos, vol. 4, no. 2, pp. 303-309, 1994.

[6] Yu. L. Maistrenko et al., "Bifurcations of attracting cycles from timedelayed Chua's circuit," Int. J. Bifurc. Chaos, vol. 5, no. 3, pp. 653-671, 1995.

[7] E. A. Hosny and M. I. Sobhy, "Analysis of chaotic behavior in lumpeddistributed circuits applied to the time-delayed Chua's circuit," IEEE Trans. Circuits Syst., vol. 41, pp. 915-918, Dec. 1994.

[8] D. Winklestein, B. B. Steer, and R. Pomerleau, "Simulation of arbitrary transmission line networks with nonlinear termination," IEEE Trans. Circuits Syst., vol. 38, pp. 418-422, Apr. 1991.

[9] J. R. Griffith and M. S. Nakhla, "Time-domain analysis of distributed networks," in Proc. IEEE ISCAS, 1991, pp. 886-890.

[10] F. H. Branin, Jr., "Transient analysis of lossless transmission line," Proc. IEEE, vol. 55, pp. 2012-2013, Nov. 1967.

[11] F. Y. Chang, "Waveform relaxation analysis of RLCG transmission lines," IEEE Trans. Circuits Syst., vol. 37, pp. 1394-1415, Nov. 1990.

[12] _ , "Transient analysis of lossy transmission lines with arbitrary initial potential and current distributions," IEEE Trans. Circuits Syst., vol. 39, pp. 180-198, May 1992.

[13] T. Nishi and K. Kouchi, "On the Padé approximation for the distributedconstant line," in Proc. NOLTA, 1994, pp. 21-24. 